\title{
The Manchester Coded Data Based OFDM (MCOFDM)
}

\section{Igor Mazića , Mirjana Bonkovićb , Anamaria Bjelopera ${ }^{a}$}

This paper shows a new DWT based OFDM algorithm which significantly simplifies signal processing in the transmitter and receiver. Unlike conventional DWT based OFDM a new algorithm does not use digital modulation of subcarriers either IDWT in the transmitter. The output signal from the transmitter is formed by summing the signals on the individual subchannels, encoded with the Manchester code and sampled at appropriate frequencies. In the receiver, the channel signal and data is reconstructed using DWT and Haar wavelet. Although the signal transmission is achieved using amplitude modulation, the paper shows that BER performance is commensurable to BPSK or DMWT based OFDM in the presence of AWGN.

\section{KEY WORDS}

$\sim$ Manchester code

$\sim$ Haar wavelet

$\sim$ OFDM

\author{
a. University of Dubrovnik, Department of Electronics, Dubrovnik, Croatia \\ e-mail: igor.mazic@unidu.hr \\ b. University of Split, Faculty of Electrical Engineering, Mechanical Engineering and \\ Naval Architecture \\ e-mail: mirjana@fesb.hr \\ doi: 10.7225/toms.v07.n02.005 \\ This work is licensed under (cc) BY
}

\section{INTRODUCTION}

Although the advantages of DWT based OFDM in comparison to FFT based OFDM are well known and the performance of such systems measured in the channels with different types of noise and digital modulation of the subcarriers as well, detailed insight into the applied transformations is usually missed. Hence, possible optimization of such systems resulting with improved performance and/or efficiency of the hardware components is omitted too. In this paper, the attention is oriented to the well-known data coding procedure (a Manchester code) which revealed very interesting properties if the Haar wavelet transformation is performed under specific conditions. This way, a completely new, digitally coded (OFDM) subcarrier transmission appears across AM modulation scheme, with improved performance regarding the complexity of used hardware and BER performance commensurable to BPSK or DMWT based OFDM in the presence of AWGN.

To present the idea clearly, the paper is organized as follows. In Section II which follows, the main characteristics of the OFDM system are described. Section III describes the effects of specific DWT compression applied to the digital signal represented using Manchester code. In Sections IV and V, the proposed procedure is used for the new, compressed based OFDM method and the effect on the hardware requirements reduction is presented. The standard analysis of such systems in terms of BER performance is presented in Section VI, and the possible further improvements of such systems regarding the signal type transmission across the subcarriers. Section VII concludes the paper.

\section{ORTHOGONAL FREQUENCY MULTICARRIER MODULATION TECHNIQUES}

Orthogonal frequency division multiplexing (OFDM) is a multicarrier modulation technique which divides the 
available spectrum into a number of parallel subcarriers where each subcarrier is modulated by a low rate data stream at the different carrier frequency. Each subcarrier is modulated with a conventional modulation scheme such as QAM (quadrature amplitude modulation) or PSK (phase shift keying) at a low symbol rate, maintaining total data rates similar to conventional single carrier modulation schemes in the same bandwidth. Multicarrier modulation schemes divide the input data into bands upon which modulation is performed and multiplexed into the channel at different carrier frequencies so that information is transmitted on each of the subcarriers. Individual subcarriers are orthogonal to each other, which allows overlapping of subcarriers because the orthogonality ensures the separation of subcarriers in the receiver. This approach suppresses channel distortion and improves the spectral efficiency considering to other types of systems like Frequency Division Multiple Access, where no spectral overlap of carriers is allowed (Dawalbait and Bilal, 2016).

The OFDM is used in many applications such as IEEE 802.11 wireless standard, Cellular radios, GSTN (General Switched Telephone Network), DAB (Digital Audio Broadcasting), DVB-T (Terrestrial Digital Video Broadcasting), HDTV broadcasting, DSL and ADSL modems and HIPERLAN type II (High Performance Local Area Network) (Hara and Prasad, 2003; Li and Stüber, 2006).

The OFDM systems are very sensitive to phase noise and CFO (carrier frequency offset - the frequency drifts in oscillators, radio propagation, and Doppler shift). These phase distortions destroy the subcarriers' orthogonality and lead to common phase error and $\mathrm{ICI}$ (inter carrier interferences). Many approaches have been proposed to estimate and eliminate phase noise effects in OFDM systems over frequency selective channels. These techniques can be divided into the frequency domain and time domain approaches (Septier and Delignon, 2011).

The FFT based OFDM uses IFFT in the transmitter for simultaneous parallel multiplexing and to generate orthogonal subcarriers and FFT in the receiver for separation of orthogonal subcarriers. The main disadvantage of FFT based OFDM is using a rectangular window which creates high side lobes. Moreover, the pulse shaping function used to modulate each subcarrier extends to infinity in the frequency domain. This leads to high interference and lower performance levels (Lakshmanan and Nikookar, 2006). Inter symbol interference (ISI) and inter carrier interference can be avoided by adding a cyclic prefix (CP) to the beginning of the OFDM symbol. A CP is added to every symbol to combat the delay spread introduced by multipath channel and the length of CP must be at least the same size as the expected channel delay spread. It can reduce the spectrum efficiency for 20-25 \% (Lakshmanan and Nikookar, 2006) or more.

The DWT based OFDM system uses IDWT in the transmitter for simultaneous parallel multiplexing and to generate orthogonal subcarriers and DWT in the receiver for separation of orthogonal subcarriers. Both systems FFT and DWT based OFDM share many similarities in terms of their functions (orthogonal subcarriers and overlapping of which makes them spectrally efficient). But, they have also some distinctive features that make them different from each other. Wavelet transform provides the time-frequency representation of signals, whereas discrete Fourier transform gives only the frequency representation. Therefore, in FFT based OFDM system subcarriers overlap in the frequency domain, whereas in DWT based OFDM system subcarriers overlap in both time and frequency domain. DWT based OFDM symbols fulfill the property of double shift orthogonally and therefore, their overlapping does not cause ISI and does not require CP. This feature makes DWT based OFDM system more spectral efficient compared to FFT based OFDM system (Oksuz et al., 2016; Parihar and Singh, 2017). Furthermore, their basic functions are different. Fourier transform decomposes a signal into sinusoidal basis functions of different frequencies whereas wavelet transform decomposes a signal into a set of mutually orthogonal wavelet basis functions. Unlike Fourier transform, wavelet transform has the ability to analyze the local properties of the input signal such as edges or transients. Wavelet based OFDM is simple to implement with less complexity than FFT based OFDM.

Many authors use computer simulations to analyze and compare FFT and DWT based OFDM system using different modulation techniques such as M-PSK and M-QAM considering to three distortion channels: AWGN-additive white Gaussian noise (Dawalbait and Bilal, 2016; Shadab and Madhav, 2015; Oksuz et al., 2016; Parihar and Singh, 2017; Chauhan and Tyagi, 2014; Goyani and Shah, 2015; Ghaith et al., 2013; Gupta and Tiwari. 2013; Kattoush, Mahmoudb and Nihadc, 2010.), Rayleigh (Dawalbait and Bilal, 2016; Shadab and Madhav, 2015; Oksuz et al., 2016; Goyani and Shah, 2015; Kumbasar and Kucur, 2012; Gupta and Tiwari, 2013) and Rician fading channel (Dawalbait and Bilal, 2016; Shadab and Madhav, 2015; Kattoush, Mahmoudb and Nihadc, 2010). The BER (bit error rate) as a function of the energy per bit to noise ratio Eb/NO and signal to noise ratio SNR have been used for performance comparison. The results of these computer simulations clearly show that DWT based OFDM outperformed the FFT based OFDM system in all three distortion channels in terms of BER. Adaptive modulation technique can additionally improve BER performance (Ali, 2015). In the comparison of BER performance of FFT and DWT based OFDM system, wavelet based OFDM system gives $1 \mathrm{~dB}$ improvement in AWGN channel and 2-8 dB improvement in Rayleigh fading channel (Gupta and Tiwari. 2013).

Considering to the CFO, DWT based OFDM also outperforms FFT based OFDM using DPSK (Sonu and Priya, 2016) and M-PSK (Jhingan and Kansal, 2016) modulations in the presence of AWGN. Also in Lee and Ryu (2018) it is shown that all systems of waveletOFDM considering various wavelets confirm PAPR (peak to average power ratio) performance lower than conventional OFDM. 
The DMWT (Discrete Multiwavelet Transform) based OFDM achieves much lower bit error rates and better performance than FFT-OFDM and DWT-OFDM in the presence of AWGN. To have a $\mathrm{BER}=10-3$ DMWT based OFDM requires $10 \mathrm{~dB}$ SNR, while DWTOFDM requires $16.5 \mathrm{~dB}$ and FFT-OFDM requires $31.5 \mathrm{~dB}$ SNR (Kattoush, Mahmoudb and Nihadc, 2010).

Comparing the commonly used wavelets (Haar, Daubechies, Biorthogonal...) in DWT based OFDM, computer simulations show that Haar and Biorthogonal wavelet have better performance in terms of BER (Veena et al., 2014; Bodhe, Joshi and Narkhede, 2012; Gupta and Tiwari, 2013).

In typical DWT based OFDM transmitter, a high data rate stream passes through encoder which is consisted of convolutional encoder followed by interleaver. This encoding technique is used for error correcting and it is used not only in OFDM systems. After encoding, the data stream is brought to the serial to parallel converter which converts the high rate serial data into lower rate parallel data, and then the data is brought to the modulator. In modulator, the subcarriers are modulated by the parallel data streams using PSK or QAM digital modulation technique, and then the signals proceed to IDWT. After IDWT the carrier mapping is used to raise subcarriers frequency to the main carrier frequency.

This paper introduces a new DWT based OFDM algorithm which significantly simplifies signal processing in the transmitter and receiver. Unlike conventional DWT based OFDM, a new algorithm does not use digital modulation of subcarriers either IDWT in the transmitter. IDWT is not used at all, but DWT is used in the receiver for signal and data reconstruction. It is achieved using appropriate data sampling and coding. A Manchester coded data stream in subchannels and their sum is a method to compose OFDM signal in the transmitter and Haar wavelet is used for signal and data reconstruction in receiver's DWT.

\section{ORTHOGONAL CODING USING MANCHESTER CODE AND HAAR WAVELET}

One of the most important assumptions in OFDM systems is a strict relation among carrier frequencies i.e.

$f_{n}=f_{c}+\Delta f^{*} n$

where $f_{c}$ is a carrier frequency, $\Delta f$ is a symbol frequency and $n$ is an integer. Therefore, the carriers are orthogonal to each other and packed tight enabling the overlapping of the subcarrier and increasing the spectral efficiency of the transmitted signal. However, considering that DWT OFDM outperforms FFT based OFDM due to double shift orthogonality, we were curious if the multiplexing (OFDM) procedure as a whole can be improved. Having a deeper insight into the specific DWT transformation over the Manchester coded data, an interesting property appeared which prompted the idea of generating the subcarriers data using DWT. The step by step procedure is minutely described in the text which follows, and the properties of the specific coded data and how it can be used for subcarrier definition are explained.

Generally, the DWT decomposition scheme is shown in Figure 1. Input signal (data) $x(n)$ is passed through half band low $(\mathrm{L})$ and half band high $(\mathrm{H})$ pass filters resulting with the filtered sequence of data $g(n)$ and $f(n)$ respectively. If the Haar wavelet is used then the filter's coefficients are $I(n)=[1 / \sqrt{ } 2,1 / \sqrt{ } 2]$ for $L$ filter and $h(n)=[-1 / \sqrt{ } 2,1 / \sqrt{ } 2]$ for $\mathrm{H}$ filter. After the downsampling procedure, the output sequences $g(2 n)$ or $C D$ (detail coefficients) and $f(2 n)$ or $C A$ (approximation coefficients) are obtained.

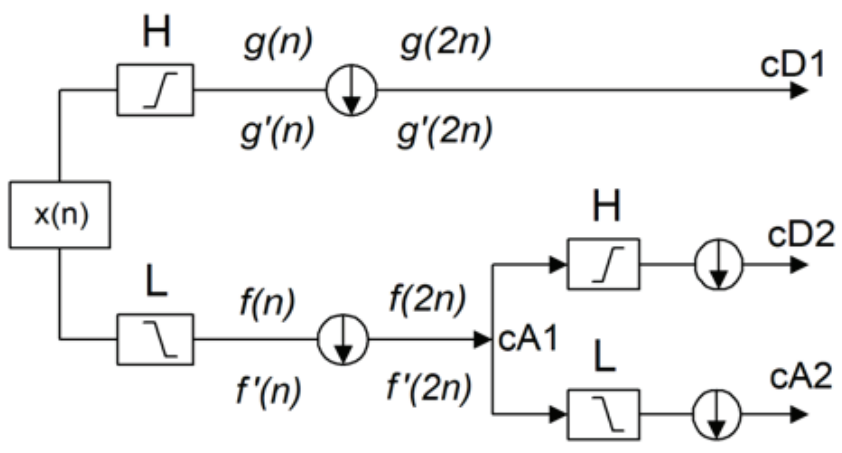

Figure 1.

A two-level discrete wavelet decomposition scheme.

Lemma1: If the discrete Haar wavelet function (Figure 2a)

$\psi(n)=\left\{\begin{array}{cc}1 & n=0 \\ -1 & n=1 \\ 0 & \text { otherwise }\end{array}\right.$

is applied for decomposition of the input function

$x(n)=\left\{\begin{array}{cc}1 & 0 \leq n<N \\ -1 & N \leq n<2 N \\ 0 & \text { otherwise }\end{array}\right.$

where $x(n)$ represents the dilated Haar wavelet function for dilatation factor $N$, where $N \varepsilon N$ (Figure 2c), then detail coefficients are:

$c D_{1}=0$

if the dilatation factor $N$ is even. The same is worth for $x(-n)$ function. 


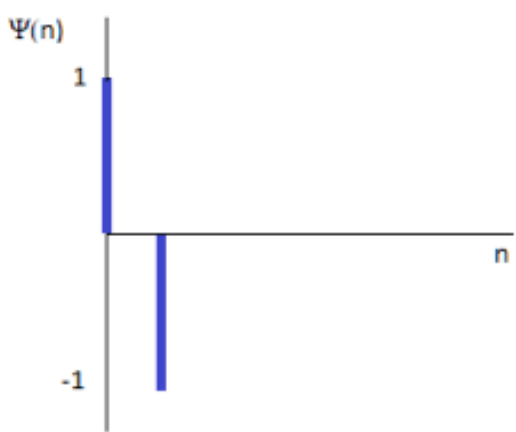

a)

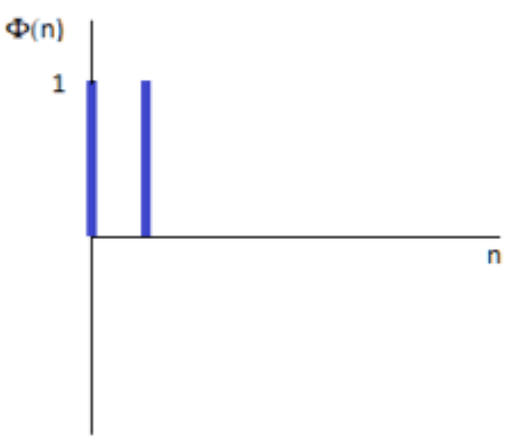

b)

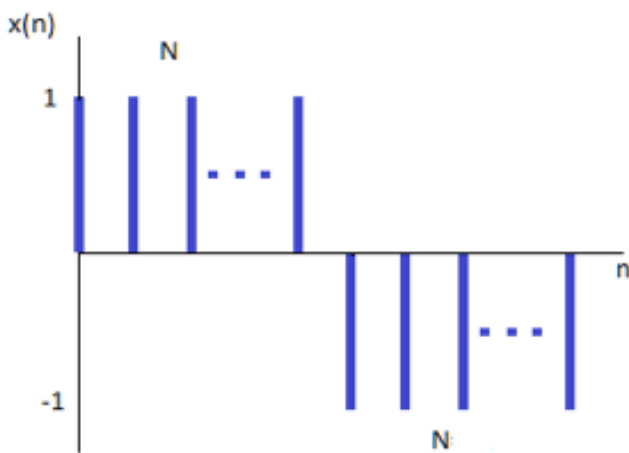

c)

Figure 2.

Haar wavelet (a), Haar scaling function (b) and $x(n)$ - dilated Haar wavelet for factor $N(c)$.

Discussion: Considering the DWT decomposition scheme, convolutions $g(n)=x(n)^{*} \psi(n)$ and $g^{\prime}(n)=x(-n)^{*} \psi(n)$ for different $N$ are shown in Table 1. After downsampling, the detail coefficients $c D_{1}=g(2 n)$ or $c D_{1}^{\prime}=g^{\prime}(2 n)$ appear. In Table $1, g(2 n)$ and $g^{\prime}(2 n)$ are marked with orange color. It can be observed that $g(2 n)$ and $g^{\prime}(2 n)$ have all zeros if $N$ is even.

Table 1.

Convolution results of discrete functions $x(n)$ and $\psi(n)$ for different $N$.

\begin{tabular}{l} 
N \\
\hline 1 \\
\hline 2 \\
\hline 3 \\
\hline 4 \\
\hline 5 \\
\hline 6
\end{tabular}

N

1

2

3

4

5

6 $g(n)=x(n) * \psi(n)$

$1,-2,1$

$1,0,-2,0,1$

$1,0,0,-2,0,0,1$

$1,0,0,0,-2,0,0,0,1$

$1,0,0,0,0,-2,0,0,0,0,1$

$1,0,0,0,0,0,-2,0,0,0,0,0,1$ $g^{\prime}(\mathbf{n})=x(-n) * \Psi(n)$

$1,2,1$

$-1,0,2,0,-1$

$-1,0,0,2,0,0,-1$

$-1,0,0,0,2,0,0,0,-1$

$-1,0,0,0,0,2,0,0,0,0,-1$

$-1,0,0,0,0,0,2,0,0,0,0,0,-1$
Lemma 2: If the discrete Haar scaling function (Figure $2 \mathrm{~b}$ )

$\Phi(n)=\left\{\begin{array}{cc}1 & n=0 \\ 1 & n=1 \\ 0 & \text { otherwise }\end{array}\right.$

is applied for decomposition of the function $x(n)$ represented by the dilated Haar wavelet function (Figure 2c), then approximation coefficients are:

$q \cdot c A_{1}=x(2 n)$

if the dilatation factor $N$ is even. The same is worth for $x(-n)$ function.
Discussion: Considering the DWT decomposition scheme, convolutions $f(n)=x(n) * \Phi(n)$ and $f^{\prime}(n)=x(-n) * \Phi(n)$ for different $N$ are shown in Table 2. After downsampling the approximation coefficients $c A_{1}=f(2 n)$ or $c A_{1}^{\prime}=f^{\prime}(2 n)$ appear. In Table 2, $f(2 n)$ and $f^{\prime}(2 n)$ are marked with orange color. It can be noted that $1 / 2 f(2 n)=x(2 n)$ if $N$ is even.

Using the results from Table 1. and Table 2. it is obvious that detail coefficients $C D_{m}$ will all be zero if $N=2^{m}$. In general, for $m$ decomposition level and $N=2 m$ the approximation and detail coefficients are:

$q \cdot c A_{m}=x\left(2^{m} \cdot n\right) \quad$ and $\quad c D_{1,2,3 \ldots m}=0$ 
Table 2.

Convolution results of discrete functions $x(n)$ and $\Phi(n)$ for different $N$.

\begin{tabular}{lcc}
$\mathbf{N}$ & $\mathbf{f}(\mathbf{n})=\mathbf{x}(\mathbf{n}) * \boldsymbol{\Phi}(\mathbf{n})$ & $\mathbf{f}^{\prime}(\mathbf{n})=\mathbf{x}(-\mathbf{n}) * \boldsymbol{\Phi}(\mathbf{n})$ \\
\hline 1 & $1,0,-1$ & $-1,0,1$ \\
\hline 2 & $1,2,0,-2,-1$ & $-1,-2,0,2,1$ \\
\hline 3 & $1,2,2,0,-2,-2,-1$ & $-1,-2,-2,0,2,2,1$ \\
\hline 4 & $1,2,2,2,0,-2,-2,-2,-1$ & $-1,-2,-2,-2,0,2,2,2,1$ \\
\hline 6 & $1,2,2,2,2,0,-2,-2,-2,-2,-1$ & $-1,-2,-2,-2,-2,0,2,2,2,2,1$ \\
\hline & $1,2,2,2,2,2,0,-2,-2,-2,-2,-2,-1$ & $-1,-2,-2,-2,-2,-2,0,2,2,2,2,2,1$
\end{tabular}

where $\mathrm{q}$ is a normalized factor.

Lemma 3: If the Haar DWT decomposition is applied to the function

$s(n)=\Sigma_{i} \xi_{i} \cdot x\left(n-i \cdot 2^{m+1}\right)$

composed of shifted $x(n)$ and/or $-x(n)$ i.e. $\xi_{i} \varepsilon\{1,-1\}$ (Manchester coded random binary data sequence) (Figure 3 ), where $m$ is decomposition level, then detail coefficients will all be zero and approximation coefficients exist at $m$ decomposition level.

Discussion: As the signal definition (the number of samples could be expressed as an exponent of base 2) meets the Lemma 1 requirement, the detail coefficients of the next decomposition level are zero. From Lemma 2, approximation coefficients of the next decomposition level are scaled values of the previous level (upsampled) approximation coefficients. Therefore, their values are the same and the number of samples could be expressed as an exponent of base 2 so again, according to
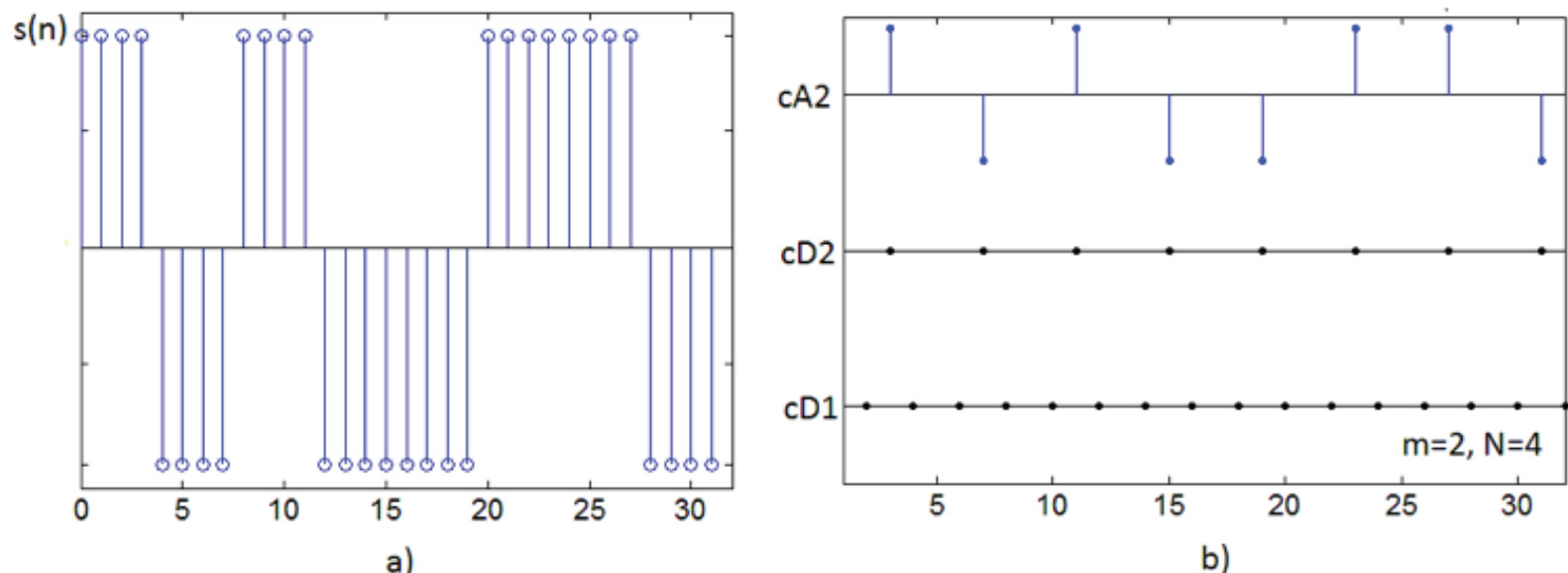

b)
Lemma 1, their detailed coefficients on the next decomposition level are zero. Consequently, the signal decomposition results with approximation coefficients which exist at only one $(m)$ decomposition level.

The process is illustrated in Figure 3 . An example of $s(n)$, defined for $m=2, N=2^{m}=4$ and composed of $x(n), x(n-8),-x(n-$ 16), $x(n-24)$ is shown in Figure 3a, whereas its two-level DWT decomposition is shown in Figure $3 \mathrm{~b}$. It can be noted that $c A_{2}$ represents the narrowest version of $s(n)$ and in general, $c A_{m}$ will represent the narrowest version of $s(n)$ if $N=2^{m}$.

However, if the decomposition level increases by $k \varepsilon N$ with the same $N$, where $N=2^{m-k}$ (in Figure 1 input sequence for 3rd decomposition level is $C A_{2}$ ) the result for $C D^{m+1}$ and $C A^{m+1}$ would correspond to the every even and negative values shown in Tables 1. and 2. for $N=1$. Of course, it is necessary to multiply by the normalizing factor in order to obtain the exact results in regard to the data in the tables. The results are shown in Figure $4 \mathrm{a}$ for $k=1, m=2, N=4$ and Figure $4 b$ for $k=2, m=2, N=4$.

Figure 3.

Input sequence $s(n)(a)$, two-level DWT decomposition of $s(n)(b)$. 


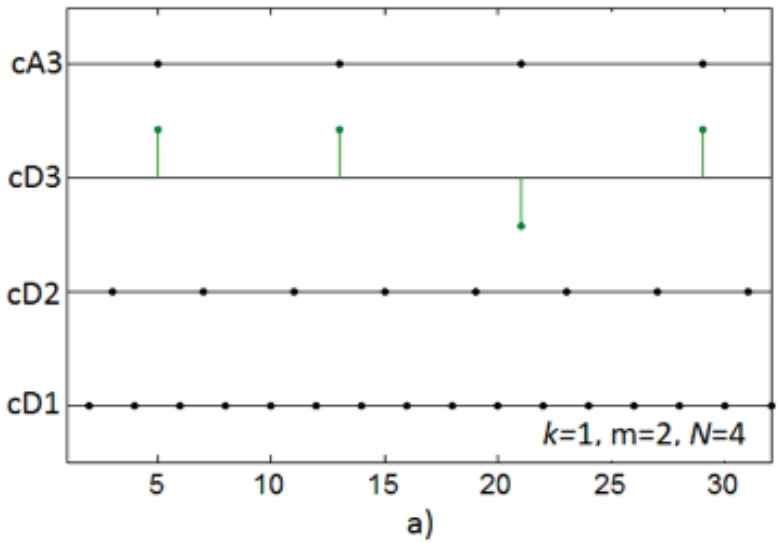

Figure 4.

Three-level decomposition of $s(n)(a)$, four-level decomposition of $s(n)(b)$.

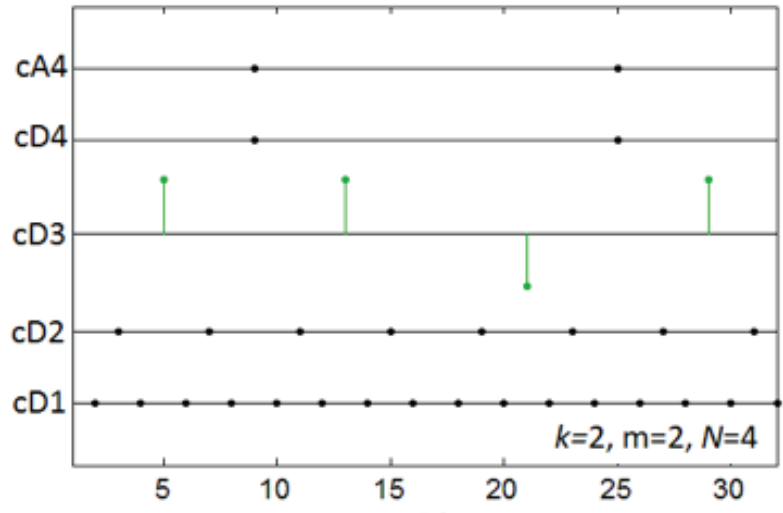

b)
It can be noted that sign of the detail coefficients at the $m+1$ level (in above examples $c D_{3}$ ) represents coefficients $\xi_{i} \varepsilon\{1,-1\}$ of the function $s(n)$. Furthermore, all the energy is concentrated in just one filter bank i.e. in just one subchannel. All these observations expressed through Lemma 1 and Lemma 2 found the base for orthogonal coding using the Manchester code and DWT decomposition with Haar wavelet explained in the next section.

\section{MCOFDM SIGNAL COMPOSITION}

Let us suppose that input binary data $u_{1}=(1,1,0,1), u_{2}=(0$, $1,1,0,1,0,1,1)$ and $u_{3}=(0,1,1,1,1,0,0,1,0,1,0,1,0,0,1,1)$ in three subchannels are set (the number of data in subchannels must increase with power of two). All the data in subchannels $u_{n}$ are coded by the Manchester coding scheme but with different clock frequency $f_{n}=f_{1} \cdot 2^{n-1}$ where $\mathrm{n}$ is a subchannel number. After Manchester coding, all encoded signals in subchannels $s n(t)$ (every $s n(t)$ correspond to un) are summed and the result is $s(t)$. The general algorithm of MCOFDM signal composition on the transmitter side is shown in Figure 5 and in general $s(t)$ is defined as:

$s(t)=\sum_{n=1} \sum_{i=0} \psi\left(\frac{t-i}{2^{1-n}}\right) \cdot\left[2 \cdot u_{n, i}-1\right]$

where $n$ is subchannel number, $i$ is data ordinal number in subchannel $u_{n}$ and $\psi(t)$ is continuous Haar wavelet defined as: $\psi(t)=\left\{\begin{array}{cc}1 & 0 \leq t<1 / 2 \\ -1 & 1 / 2 \leq t<1 \\ 0 & \text { otherwise }\end{array}\right.$

According to the above defined data $u_{1^{\prime}} u_{2}$ and $u_{3^{\prime}}$ appropriate signals in subchannels $s_{1}(t), s_{2}(t), s_{3}(t)$ and theirs sum $s(t)$ are shown in Figure 6.

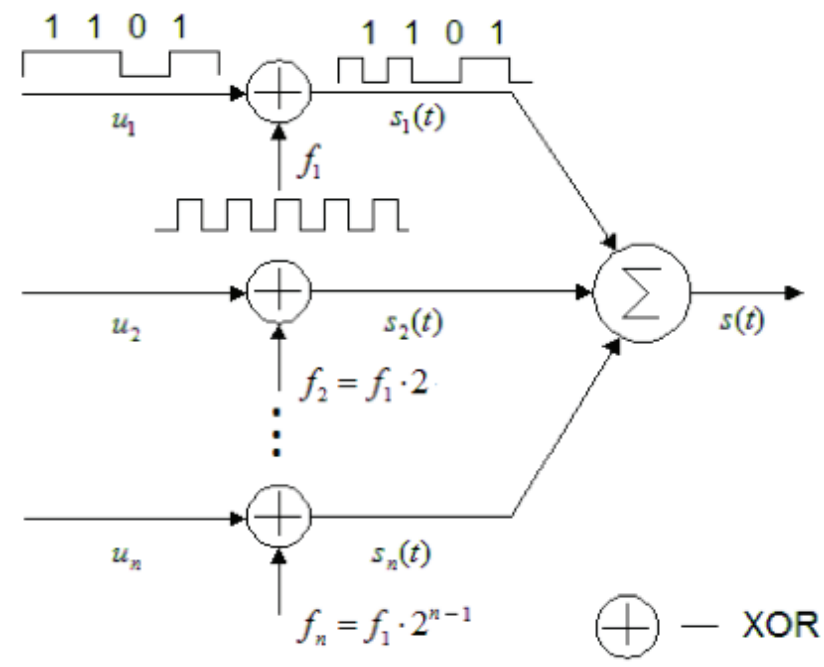

Figure 5.

MCOFDM signal composition. 

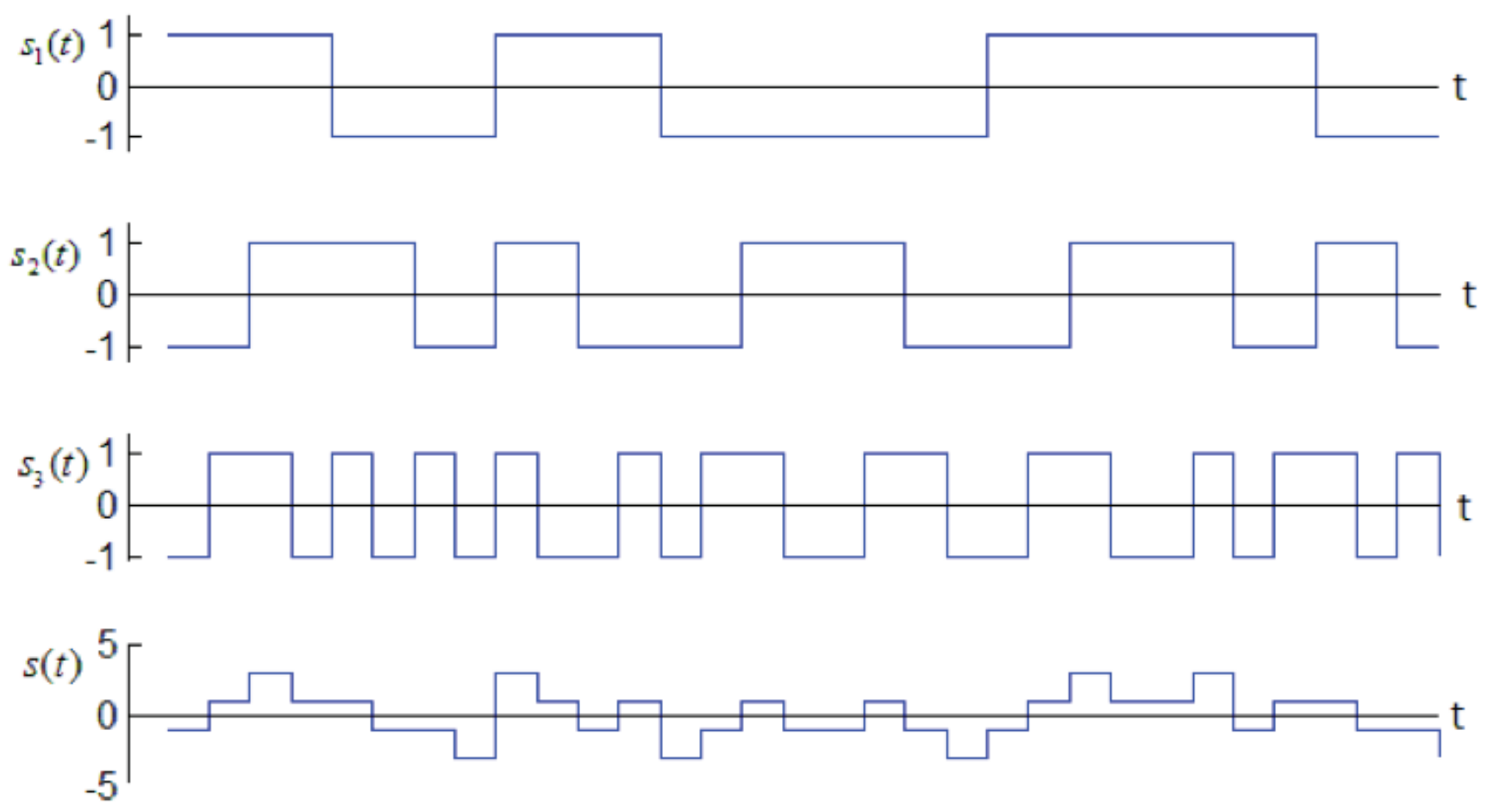

Figure 6.

Subchannel signals $s_{1}(t), s_{2}(t), s_{3}(t)$ and theirs sum $s(t)$.

\section{MCOFDM SIGNAL DECOMPOSITION - BINARY DATA RECONSTRUCTION}

As a standard OFDM procedure on the receiver side, the DWT decomposition scheme (see Figure 1 for two-level

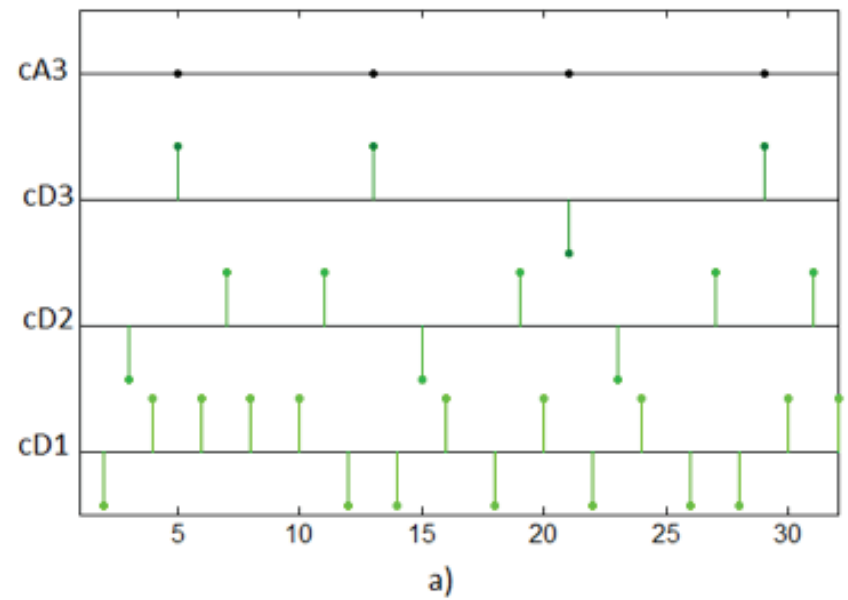

decomposition and some input signal $x(t)$ has to be performed. If the sampling frequency is $F_{s}=f_{n}=f_{1} \cdot 2^{n}$ (in this example $\left.F_{s}=f_{1} \cdot 8, n=3\right)$ and the input signal is $s(t)=s_{1}(t)+s_{2}(t)+s_{3}(t)$, the output decomposition coefficients for 3-level DWT decomposition are shown in Figure $7 a$.

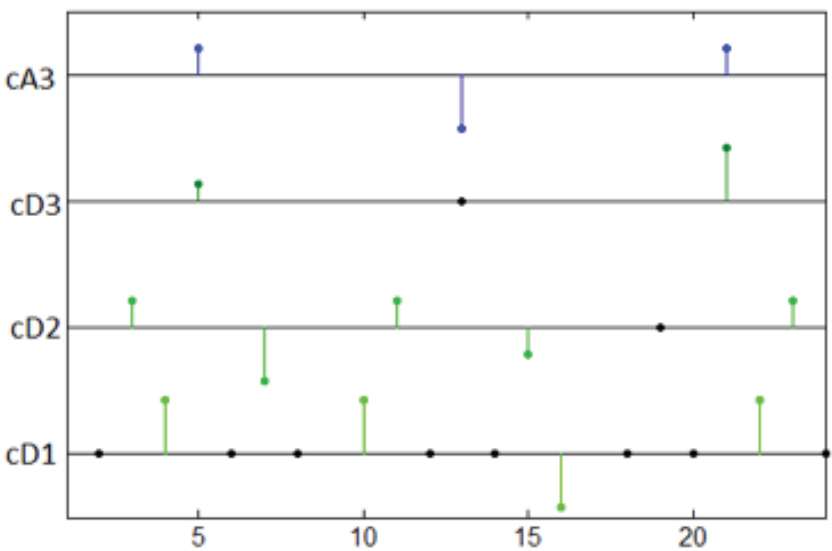

b)

Figure 7.

Decomposition coefficients of $s(t)$ for sampling frequency $F_{s}=8 f_{1}(a)$, decomposition coefficients of $u_{1}(t)$ for sampling frequency $F_{s}=6 f_{1}(b)$. 
As shown in Figure 7a the output decomposition coefficients $C D 3, C D 2$ and $C D 1$ correspond to input data $u_{1}, u_{2}$ and $u_{3}$ respectively (the positive values correspond to the logical ones and the negative to the logical zeros). If data in subchannels are not coded by Manchester code or $F_{s} \neq f_{i} \cdot 2^{n}$ or $f_{n} \neq f_{i} \cdot 2^{n}$ the energy will not concentrate in only one subchannel and the reconstruction will not be possible in the described way. The mentioned case is illustrated in Figure $7 \mathrm{~b}$ where the binary input data is $u_{1}=(1,1,0,1)$ which correspond to $s(t)=s_{1}(t)$ and $F_{s}=6$ - $f_{1}$. According to above data reconstruction algorithm, it is not possible to obtain input data $u_{1}$ directly from decomposition coefficients (note that zeros in cD1 are not defined) and the energy is dispersed through the subchannels causing the interference in the adjacent subchannels. Moreover, even in the case of jitter $\left(u_{1}=(1,1,0,1)\right)$, but rectangular pulses after Manchester coding on the receiver side do not have the same width, see Figure 8a) reconstruction is possible in that subchannel (see $c D 3$ in Figure $8 b)$, but the energy is also dispersed through the subchannels causing the interference in the adjacent subchannels.

\section{BER PERFORMANCE}

The BER performances for MCOFDM over AWGN channel were gained using Matlab programming according to the scheme on Figure 9.

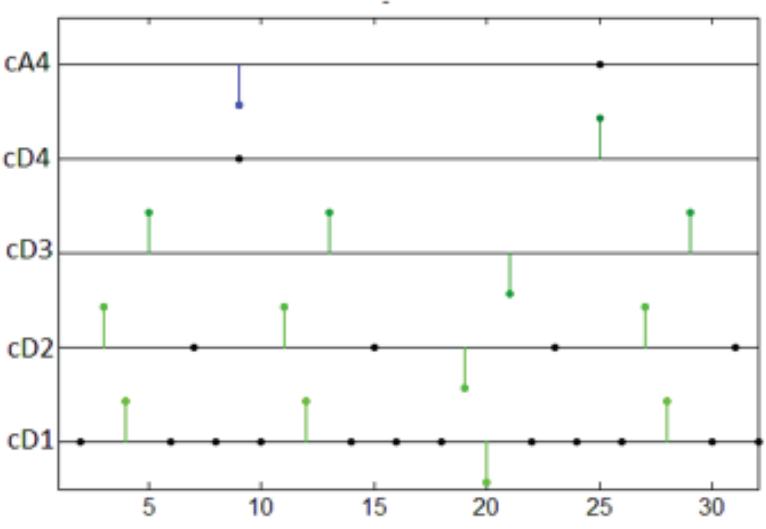

b)

Figure 8.

Jitter - rectangular pulses do not have the same width (a), decomposition coefficients in case of jitter (b).

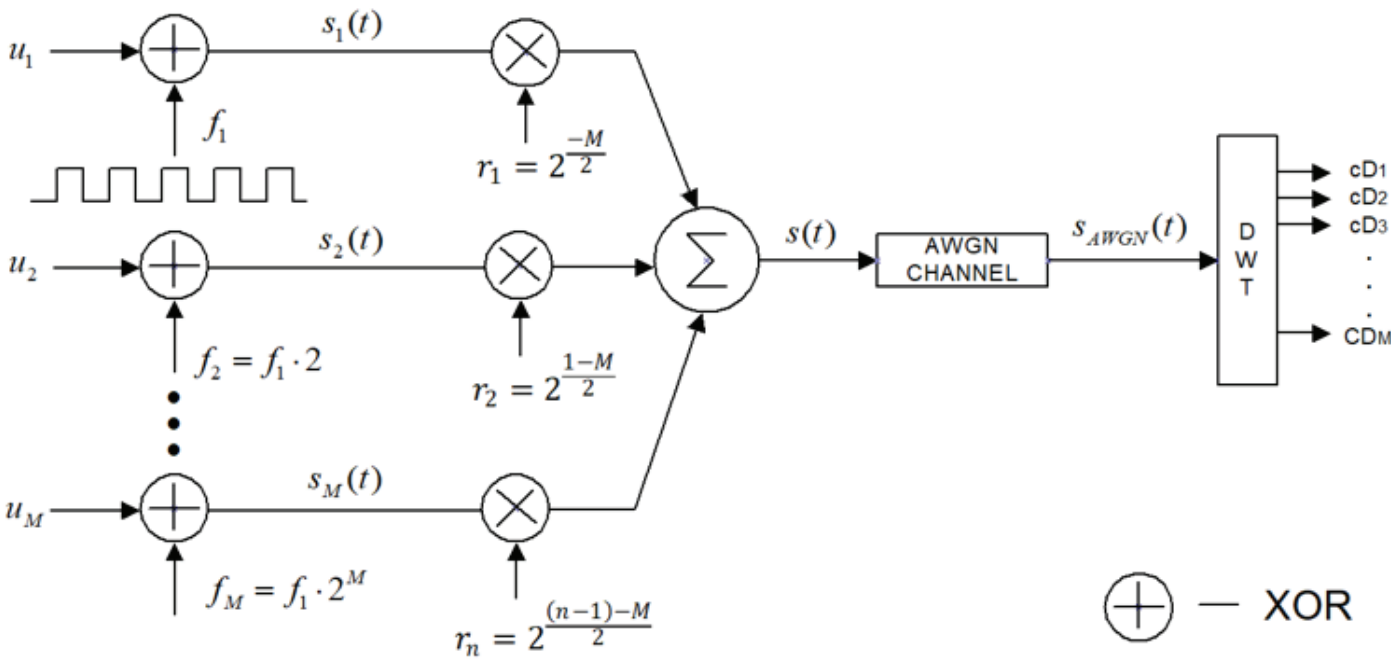

Figure 9.

MCOFDM scheme. 
Two computer simulations were carried out. Both simulations use 8 subchannels $(M=8)$ with the total of 511 random bits (first subchannel - 1bit, second subchannel -2 bits, third subchannel -4 bits...) and 20.000 iterations are processed, every time with different input data. The first computer simulation results (normalization factors $r_{1 \ldots M}=1$ ) showed that the errors were not distributed evenly over all subchannels. The subchannels with a higher ordinal number have a greater number of errors. The blue curve in Figure 10 shows BER performance for OFDM based on Manchester code and Haar wavelet over AWGN channel without normalization.

To ensure even distribution of errors over the subchannels it is necessary to reduce the amplitude of the signal in the lower order subchannels and to increase it in the higher order subchannel. Therefore, the total power of the signal would remain the same. Hence, the expression (8) has to be overwritten by inserting a normalization factors

$r_{n}=2^{\frac{(n-1)-M}{2}}$

where $n$ is a channel number and $M$ is the total number of channels.

Finally, the OFDM signal based on orthogonal coding using the Manchester code and Haar wavelet with normalization is defined using Eq. (11):

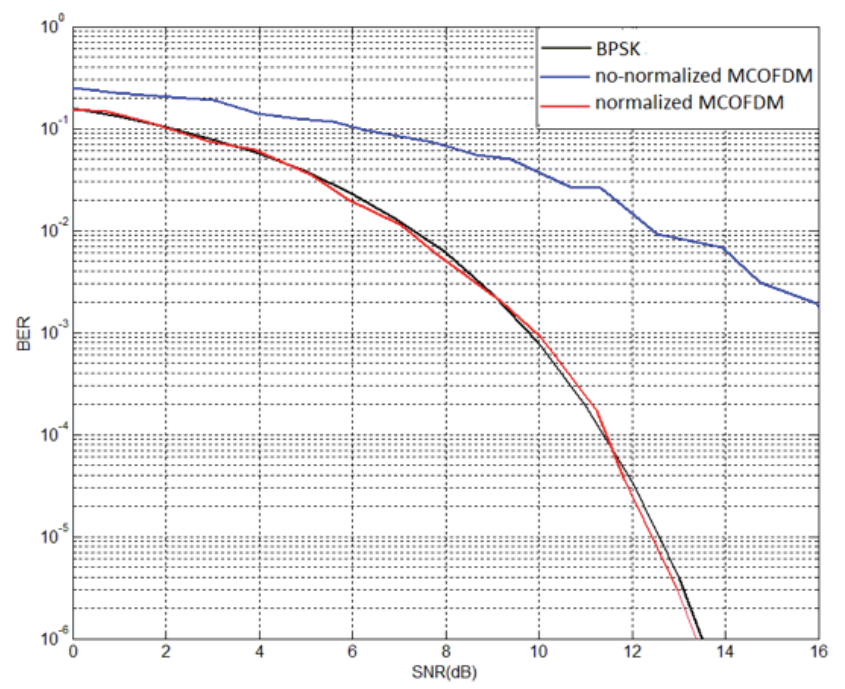

Figure 10.

BER performance over AWGN channel for BPSK, normalized and no-normalized OFDM based on Manchester code and Haar wavelet . $s(t)=\sum_{n=1} 2^{\frac{(n-1)-M}{2}} \sum_{i=0} \psi\left(\frac{t-i}{2^{1-n}}\right) \cdot\left[2 \cdot u_{n, i}-1\right]$

and the result of second computer simulation (BER performance for MCOFDM over AWGN channel with normalization) is shown in the red curve in Figure 10. For comparison purpose, the black curve shows theoretical BER performance for BPSK over AWGN channel (Soniwal, 2018).

\section{CONCLUSIONS}

The main advantage of amplitude digital modulation technique like ASK is simplicity, but it is very susceptible to noise interference due to noise affects the amplitude. Therefore, ASK is not used in OFDM and generally neither in any noise affected systems. PSK is less susceptible to errors than ASK. It occupies the same bandwidth, but a main disadvantage of PSK is more complex signal detection and recovery process. Moreover, QAM as the combination of PSK and ASK is even more complex than PSK. Although orthogonal coding uses amplitude digital modulation technique, this paper shows that BER performance of OFDM based on orthogonal coding using Manchester code and Haar wavelet are commensurable with BPSK or DMWT based OFDM in the presence of AWGN.

Furthermore, although the normalization procedure improves the BER performance, it should be noted that the decision if it should be used or not depends on the application. For example, if the raw data is transmitted over subchannels, the signals should be normalized. However, if audio and video are transmitted separately, the audio should be transmitted over lower order subchannels due to their noise insensitivity and video should be transmitted over higher order subchannels due to their larger width and higher capacity. If this situation occurs, the benefit achieved by normalization is questionable. Additionally, if the application requires equal subchannel widths, it is worth to explore the benefits of the PWT (packet wavelet transform) instead of DWT.

Finally, this paper clearly shows that a new DWT based OFDM algorithm significantly simplifies signal processing in the transmitter and receiver using no digital modulation of subcarriers in the receiver, neither IDWT in the transmitter. Although the signal transmission is achieved using amplitude modulation, the paper shows that BER performance is commensurable to BPSK or DMWT based OFDM in the presence of AWGN.

\section{REFERENCES}

Ali, W.T., 2015. Performance of Discrete Wavelet Transform Based on OFDM with Adaptive Modulation. International Journal of Engineering Research, 4(8), pp.419425. Available at:

http://dx.doi.org/10.17950/ijer/v4s8/804. 
Bodhe, R., Joshi, S., Narkhede, S., 2012. Performance Comparison of FFT and DWT based OFDM and Selection of Mother Wavelet for OFDM. International Journal of Computer Science and Information Technologies, 3(3), pp. 3993-3997.

Chauhan, C., Tyagi, A., 2014. Analysis of FFT and DWT based OFDM. International Journal Of Engineering And Computer Science, 3(8), pp. 7963-7969.

Dawalbait, E.M., Bilal K.H., 2016. Performance Analysis of OFDM System. International Journal of Engineering, Applied and Management Sciences Paradigms, 33(1), pp. 11-16.

Ghaith, A. et al., 2013. Performance analysis of the Wavelet-OFDM new scheme in AWGN channel. 2013 Third International Conference on Communications and Information Technology (ICCIT). Available at: http://dx.doi.org/10.1109/iccitechnology.2013.6579554.

Goyani, A., Shah, R., 2015. A Review - Performance comparison of conventional and wavelet-based OFDM system. International Journal of Innovative Science, Engineering \& Technology, 2(3), pp. 517-520, 2015.

Gupta, M.K. and Tiwari, S., 2013. Performance evaluation of conventional and wavelet based OFDM system. AEU - International Journal of Electronics and Communications, 67(4), pp. 348-354. Available at: http://dx.doi.org/10.1016/j.aeue.2012.10.005.

Hara, S., Prasad, R., 2003., Multicarrier Techniques for 4G Mobile Communications. Artech House.

Jhingan, A. and Kansal, L., 2016. Performance Analysis of FFT-OFDM and DWTOFDMover AWGN Channel under the Effect of CFO. Indian Journal of Science and Technology, 9(6). Available at:

http://dx.doi.org/10.17485/ijst/2016/v9i6/80047.

Kattoush, A.H., Mahmoud, W.A. and Nihad, S., 2010. The performance of multiwavelets based OFDM system under different channel conditions. Digital Signal Processing, 20(2), pp. 472-482. Available at: http://dx.doi.org/10.1016/j.dsp.2009.06.022.

Kumbasar, V. and Kucur, O., 2012. Performance comparison of wavelet based and conventional OFDM systems in multipath Rayleigh fading channels. Digital Signal Processing, 22(5), pp. 841-846. Available at: http://dx.doi.org/10.1016/j.dsp.2012.02.004.
Lakshmanan, M.K. and Nikookar, H., 2006. A Review of Wavelets for Digital Wireless Communication. Wireless Personal Communications, 37(3-4), pp.387-420. Available at: http://dx.doi.org/10.1007/s11277-006-9077-y.

Lee, J.-G. and Ryu, H.-G., 2018. Design and Comparison of Discrete Wavelet Transform Based OFDM (DWT-OFDM) System. 2018 Tenth International Conference on Ubiquitous and Future Networks (ICUFN). Available at: http://dx.doi.org/10.1109/icufn.2018.8437028.

Li, Y., Stüber, G.L., 2006. Orthogonal Frequency Division Multiplexing for Wireless Communications. Springer.

Oksuz, E., Altun, A., Ulgeri, B., Yucel, G. and Ozen, A., 2016. A Comparative Performance Analyses of FFT Based OFDM and DWT Based OFDM Systems. Journal of New Results in Science, 12, pp. 272-287.

Parihar, S.S., Singh, V., 2017. A Comparative Analysis of FFT and DWT based OFDM. International Journal of Innovative Engineering Research, 7(4), pp. 1-6.

Septier, F. and Delignon, Y., 2011. MCMC sampling for joint estimation of phase distortions and transmitted symbols in OFDM systems. Digital Signal Processing, 21(2), pp. 341-353. Available at:

http://dx.doi.org/10.1016/j.dsp.2010.10.003.

Shadab, T., Madhav, V., 2015. Performance and Analysis of BER in wavelets based OFDM system with different channel estimation. IOSR Journal of Electronics and Communication Engineering, pp. 140-155.

Soniwal, Y.K., 2018. BPSK BER v/s SNR Simulation for AWGN channel. Available at: https://www.mathworks.com/matlabcentral/fileexchange/37994-bpsk-ber-v-s-snrsimulation-for-awgn-channel.

Sonu, P.S., Priya, M., 2016. BER perfomance analysis of FFT and DWT OFDM using DPSK in the presence of CFO. International Journal of Engineering Sciences \& Research Technology, pp. 504-509.

Veena, S., Naveen, H., Cyril Prasanna Raj, P., 2014. Simulation and modeling of modified LDWT/IDWT algorithm for MB-OFDM on FPGA. International Conference on Electrical Electronics and Communications-ICEEC-21, pp. 125-131. Available at: http://www.iisthub.com/Proceeding_Archives/PA_ICEEC2014/125-131.pdf 